Palavras chave:

Weibull

Distribuição diamétrica Eucalyptus

Histórico:

Recebido 22/06/201 I

Aceito 29/04/2014

Keywords: Weibull

Diameter distribution

Eucalyptus

Correspondência: danielhbbinoti@gmail.com
Daniel Henrique Breda Binoti', Mayra Luiza Marques da Silva Binoti', Helio Garcia Leite², Luiz Marcos Silva Matos², Antonilmar Araújo Lopes da Silva ${ }^{3}$

\section{AJUSTE DA FUNÇÃO LOG-PEARSON TIPO III A DADOS DE POVOAMENTOS EQUIÂNEOS DE EUCALIPTO}

RESUMO: Objetivou-se, neste estudo, avaliar a eficiência da função log-Pearson tipo III para a descrição da estrutura diamétrica de povoamentos equiâneos, bem como propor um modelo de distribuição diamétrica utilizando a função. A modelagem realizada pela função log-Pearson tipo III foi comparada com a modelagem realizada com a função Weibull. Para isso, utilizaram-se dados de parcelas permanentes de eucalipto, localizadas na região Centro Oeste do Estado de Minas Gerais. Os modelos de distribuição de diâmetros utilizados basearam-se nos modelos proposto por Nogueira et al. (2005) e foram avaliados em função do RMSE (\%) e do coeficiente de correlação entre valores estimados e observados. Todos os ajustes apresentaram aderência aos dados pelo teste de Kolmogorov-Smirnov. Todas as equações ajustadas para a recuperação da distribuição diamétrica apresentaram coeficientes de correlação elevados (superiores a 0,9 ), podendo ser utilizadas para a modelagem da distribuição diamétrica de povoamentos de eucalipto.

\section{ADJUSTING THE LOG-PEARSON TYPE III FUNCTION TO EVEN-AGED EUCALYPTUS STANDS DATA}

ABSTRACT: The objective of this study is to evaluate the efficiency of the log-Pearson type III function to describe the diameter structure of even-aged stands, and propose a diameter distribution model using the function. The modeling function performed by the log-Pearson type III was compared with the modeling performed with the Weibull function. For this we used data from permanent plots of eucalyptus, located in the Midwestern region of Minas Gerais. Adherence to the functions of the data was confirmed by applying the Kolmogorov-Smirnov test (KS). The diameter distribution models used were based on models proposed by Nogueira et al. (2005) and were evaluated in terms of RMSE (\%) and the correlation coefficient between estimated and observed values. All fitting to the data presented adherence by the KS test. All equations fitted for the recovery of the diameter distribution showed high correlation coefficients and can be used for modeling the diameter distribution of eucalyptus. 


\section{INTRODUÇÃO}

A descrição das estimativas de frequência por classe diamétrica é realizada pela utilização de funções densidade de probabilidade $(f d p)$. Apesar da existência de diversas funções estatísticas, o enfoque de modelagem tem sido o mesmo, com predomínio de uma das formas da função Weibull (CAMPOS; LEITE, 20I3). Dentre outras características, a boa correlação apresentada por seus parâmetros com características do povoamento tem sido fundamental para a escolha dessa função, cuja forma usual está apresentada na equação I, sendo $\beta$ o parâmetro de escala $(\beta>0)$ e $\alpha$ o parâmetro de forma $(\alpha>0)$.

$f(x)=\frac{\alpha}{\beta} \cdot\left(\frac{x}{\beta}\right)^{\alpha-1} \cdot e^{\left(-\left(\frac{x}{\beta}\right)^{\alpha}\right)}$

Conforme Leite et al. (20l0), a avaliação da aplicação de diferentes funções densidade de probabilidade deve ser feita, a fim de identificar a aplicação de funções para a modelagem da distribuição diamétrica.

Uma função comumente utilizada para a modelagem da distribuição de chuvas é a função logPearson tipo III (FREITAS et al., 200I), descrita na equação 2 , sendo $\beta$ o parâmetro de escala $(\beta>0)$ e $\alpha$ o parâmetro de forma $(\alpha>0)$ e $\gamma$ o parâmetro de locação. A forma acumulativa está apresentada na equação 3.

$f(x)=\frac{1}{x|\beta| \Gamma(\alpha)} \cdot\left(\frac{\ln (x)-\gamma}{\beta}\right)^{\alpha-1} \cdot e^{\left(-\frac{\ln (x)-\gamma}{\beta}\right)}$

$f(x)=\frac{\Gamma_{\left(\frac{\ln (x)-\gamma}{\beta}\right)}(\alpha)}{\Gamma(\alpha)}$

Objetivou-se, neste estudo, avaliar a eficiência da função log-Pearson tipo III (SINGH, 1987) para a descrição da distribuição de diâmetros de povoamentos de eucalipto, bem como propor um modelo de distribuição diamétrica, utilizando essa função. Para fins comparativos, foi utilizada a modelagem da distribuição diamétrica com a função Weibull.

\section{MATERIAL E MÉTODOS}

\section{Dados}

Os dados utilizados neste estudo foram obtidos de 400 parcelas permanentes retangulares de $340 \mathrm{~m}^{2}$, instaladas em povoamentos de híbridos de Eucalyptus grandis $x$ Eucalyptus urophylla, na região Centro Oeste do Estado de Minas Gerais, Brasil. As medições de diâmetros foram efetuadas nas árvores com diâmetro a I,3 metros de altura (dap) acima de $5 \mathrm{~cm}$, nas idades médias de 28 , 40, 52, 64, 76 e 84 meses.

Os dados de dap observados em cada parcela foram agrupados em classe com amplitude de 1,0 cm. Para cada parcela, em cada medição, ajustou-se a função log-Pearson tipo III e a função Weibull. Os ajustes foram feitos em ambiente Excel com auxílio de técnicas de Visual Basic for Applications pelo método da máxima verossimilhança. Para avaliar a aderência da função aos dados, utilizou-se o teste Kolmogorov-Smirnorv (K-S) (GIBBONS; SUBHABRATA, 1992). Realizou-se a análise entre valores estimados e observados para todos os ajustes.

A construção do modelo de distribuição diamétrica baseou-se na alternativa usual de correlação dos parâmetros da fdp com características do povoamento. Conforme Nogueira et al. (2005), utilizaram-se como variáveis dependentes os parâmetros da função log-Pearson tipo III e Weibull em uma idade futura, e como variáveis independentes os parâmetros da função e características do povoamento em uma idade atual. Os modelos de distribuição diamétrica foram ajustados pelo método iterativo Quassi-Newton com a utilização do software Statistic 8 (STATSOFT, 2009).

O coeficiente de correlação entre os valores observados e estimados e a análise gráfica dos resíduos foram utilizados para avaliar a exatidão das estimativas do sistema de equações. Avaliou-se também a estatística RMSE (\%) (SIIPILEHTO, 2000). O estimador utilizado está apresentada na equação 4 , sendo $\bar{Y}$ a média da saída observada e n é o número total de dados.

$\operatorname{RMSE}(\%)=100 / \bar{Y} \cdot \sqrt{\frac{\sum_{i=1}^{n}\left(Y_{i}-\hat{Y}_{i}\right)^{2}}{n}}$

\section{RESULTADOS}

Ajustou-se a função log-Pearson tipo III e Weibull para todas as parcelas e em cada situação, sendo cada estimação comparada com a distribuição observada. Todos os ajustes resultaram em aderência aos dados pelo teste Kolmogorov-Smirnov $(P>0,0 \mathrm{I})$. Os valores médio do teste foram de 0,0526 e 0,053 para função Weibull e log-Pearson, respectivamente. Três parcelas foram escolhidas aleatoriamente e seus ajustes são apresentados na Figura I. 


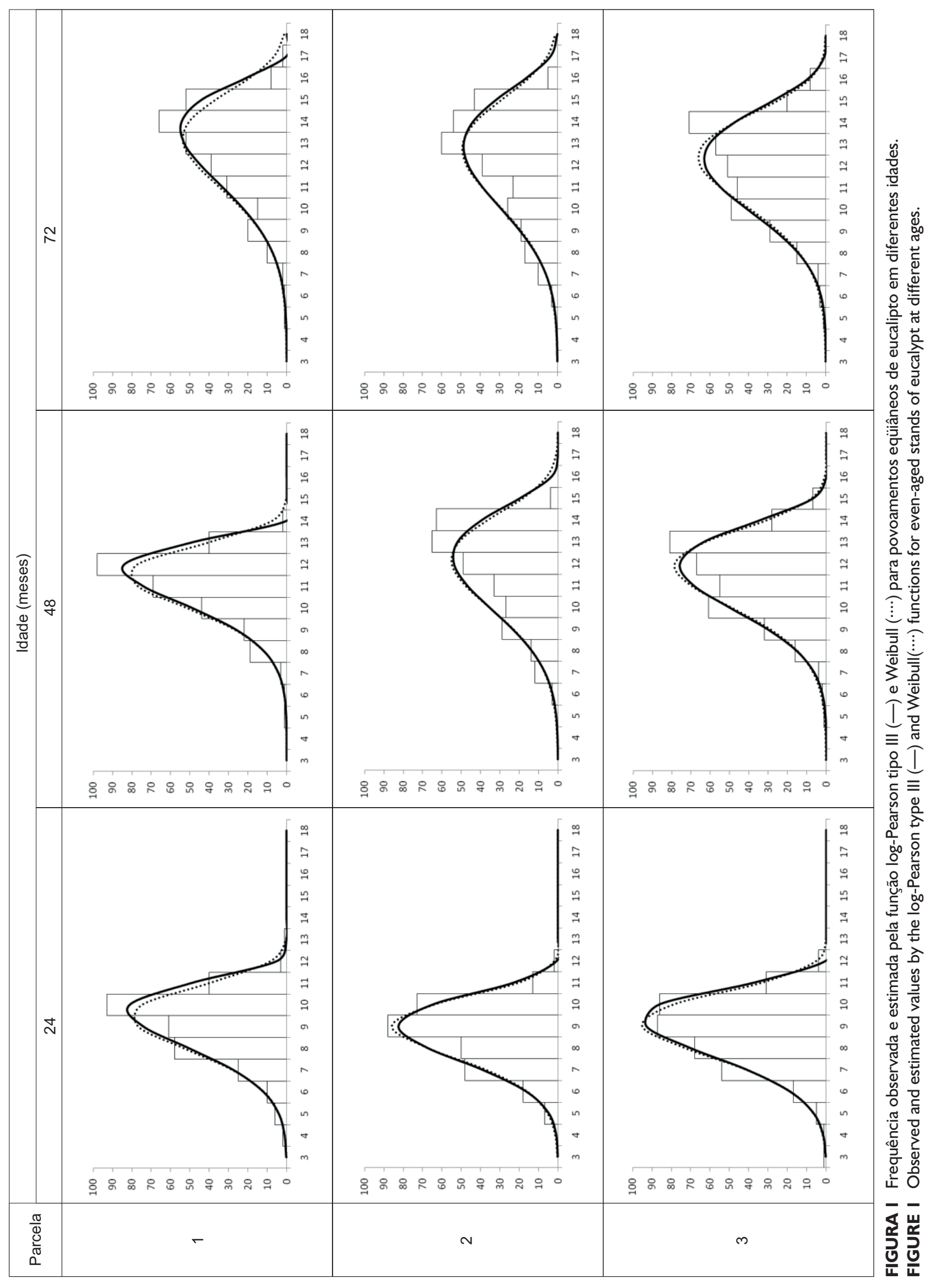


Os modelos de distribuição diamétrica ajustados com seus respectivos coeficientes de correlação e RMSE(\%) estão apresentados nas equações 5,6 , $7,8,9,10$ e $I I$, sendo $I_{1}$ e $I_{2}$ as idades atual e futura, respectivamente, em meses; $\alpha_{1}$ e $\alpha_{2}$ os parâmetros de forma da função log-Pearson tipo III, nas idades atual e futura; $\beta_{1}$ e $\beta_{2}$ os parâmetros de escala da função logPearson tipo III, nas idades atual e futura; $\gamma_{1}$ e $\gamma_{2}$ os parâmetros de locação da função log-Pearson tipo III, nas idades atual e futura; $\alpha_{1}$ e $\alpha_{2}$ os parâmetros de forma da função Weibull, nas idades atual e futura; $\beta_{1}$ e $\beta_{2}$ os parâmetros de escala da função Weibull, nas idades

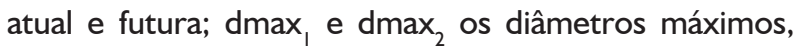
nas idades atual e futura, em $\mathrm{cm}$, dmin, e dmin 2 os diâmetros mínimos, nas idades atual e futura, em cm; e In o logaritmo neperiano.

Log-Pearson tipo III:

$\gamma_{2}=\gamma_{1} \cdot e^{\left(-411,6497 \cdot\left(L_{2}^{-0,0004}-I_{1}^{-0,0004}\right)\right)}$

$\mathrm{R}_{\mathrm{yy}}=0,9249 \quad$ RMSE (\%) $=5,569$

$\beta_{2}=\beta_{1} \cdot e^{\left(-97,0987 \cdot\left(I_{2}^{-1,8705}-I_{1}^{-1,8750}\right)\right)}$

$\mathrm{R}_{\mathrm{yy}}=0,9553 \quad$ RMSE (\%) $=19,997$

$\ln \alpha_{2}=\ln \alpha_{1} \cdot e^{\left(-421,5061 \cdot\left(I_{2}^{-0,0004}-I_{1}^{-0,0004}\right)\right)}$

$R_{\text {yy }}=0,9135 \quad$ RMSE (\%) $=40,369$

Weibull:

$\beta_{2}=\beta_{1}\left(\frac{I_{1}}{I_{2}}\right)+20,7092\left(I-\frac{I_{1}}{I_{2}}\right)$

$\mathrm{R}_{\mathrm{yy}}=0,955 \mathrm{I} \quad$ RMSE (\%) $=3,829$

$\ln \alpha_{2}=\ln \alpha_{1} \cdot e^{\left(-(0,7429) \cdot\left(1_{2}^{0,1318}-1_{1}^{0,1318}\right)\right)}$

$R_{\text {yy }}=0,9818 \quad$ RMSE (\%) $=13,356$

Povoamento:

$\operatorname{dmax}_{2}=\operatorname{dmax}_{1} \cdot\left(\frac{I_{1}}{I_{2}}\right)+25,3786 \cdot\left(I-\frac{I_{1}}{I_{2}}\right)$

$\mathrm{R}_{\mathrm{yyy}}=0,9525 \quad$ RMSE (\%) $=4,946$

$\operatorname{dmin}_{2}=d \min \cdot e_{1}\left(-(16,6040) \cdot\left(I_{2}^{1-1,1574}-I_{1}^{-1,1574}\right)\right)$

$\mathrm{R}_{\hat{y} \mathrm{y}}=0,884 \mathrm{I} \quad$ RMSE $(\%)=8,78 \mathrm{I}$

\section{DISCUSSÃO}

A aderência das funções aos dados, comprovada pelo teste K-S, demonstra a capacidade da função em descrever a estrutura diamétrica de povoamentos de eucalipto, os valores do teste foram inferiores a 0,1 . $\mathrm{Na}$ Figura I, apresenta-se o ajuste de ambas as funções logPearson tipo III e Weibull.

As equações propostas para a modelagem da distribuição diamétrica com ambas funções apresentaram valores satisfatórios de RMSE (\%), para fins de manejo florestal. Contudo, há superioridade da função Weibull em função dos menores valores de RSME (\%).

O comparativo entre as funções deve considerar, além das estatísticas demonstradas, a existência do parâmetro de locação no sistema de equações da função log-Pearson Tipo III, esse parâmetro representa um ponto de truncamento à esquerda e pode ocasionar uma melhoria nos ajustes, contudo a consistência na recuperação dos parâmetros da distribuição em uma idade futura é reduzida, sendo que cada situação deve ser avaliada individualmente, sendo influenciada, principalmente, pelo tipo de manejo e intervenções silviculturais aplicadas (CAMPOS; LEITE, 20I3).

Todas as equações que compõem os modelos de distribuição diamétrica apresentaram valores reativamente altos (superiores a 0,9) de coeficientes de correlação entre valores observados e estimados. Esse fato demonstra a capacidade de recuperação dos parâmetros das funções em idades futuras, comprovando a possibilidade de utilização destas em modelos de distribuição diamétrica de povoamentos de eucalipto.

\section{REFERÊNCIAS}

CAMPOS, J. C. C.; LEITE, H. G. Mensuração florestal: perguntas e respostas. 4. ed. Viçosa, MG: UFV, 2013. $605 \mathrm{p}$.

FREITAS, A. J. D.; SILVA, D. D. D.; PRUSKI, F. F.; PINTO, F. A.; PEREIRA, S. B.; GOMES FILHO, R. R.; TEIXEIRA, A. D. F.; BAENA, L. G. N.; MELLO, L. T. A. D.; NOVAES, L. F. D. Equações de chuvas intensas no Estado de Minas Gerais. Belo Horizonte: Companhia de Saneamento de Minas Gerais; Viçosa, MG: UFV, $200 \mathrm{I}$. $65 \mathrm{p}$.

GIBBONS, J. D.; SUBHABRATA, C. Nonparametric statistical inference. $3^{\text {rd }}$ ed. New York: M. Dekker, 1992. 544 p. (Statistics: Textbook and Monograph, 3I). 
LEITE, H. G.; BINOTI, D. H. B.; GUIMARÃES, D. P.; SILVA, M. L. M.; GARCIA, S. L. R. Avaliação do ajuste das funções Weibull e hiperbólica a dados de povoamentos de eucalipto submetidos a desbaste. Revista Árvore, Viçosa, v. 34, n. 2, p. 305- 31।, 2010.

NOGUEIRA, G. S.; LEITE, H. G.; CAMPOS, J. C. C.; CARVALHO, A. F.; SOUZA, A. L. de. Modelo de distribuição diamétrica para povoamentos de Eucalyptus sp. submetidos a desbaste. Revista Árvore, Viçosa, v. 29, n. 4, p. 579-589, 2005.
SIIPILEHTO, J. A. Comparison of two parameter prediction methods for stand structure in Finland. Silva Fennica, Vantaa, v. 34, n. 4, p. 33I-349, 2000.

SINGH, V. Hydrologic frequency modeling. Dordrecht: D. Reidel, 1987. v. I, 645 p.

STATSOFT. Statistica: data analysis software system. Version 7. Disponível em: <http://www.statsoft.com>. Acesso em: 10 dez. 2009. 